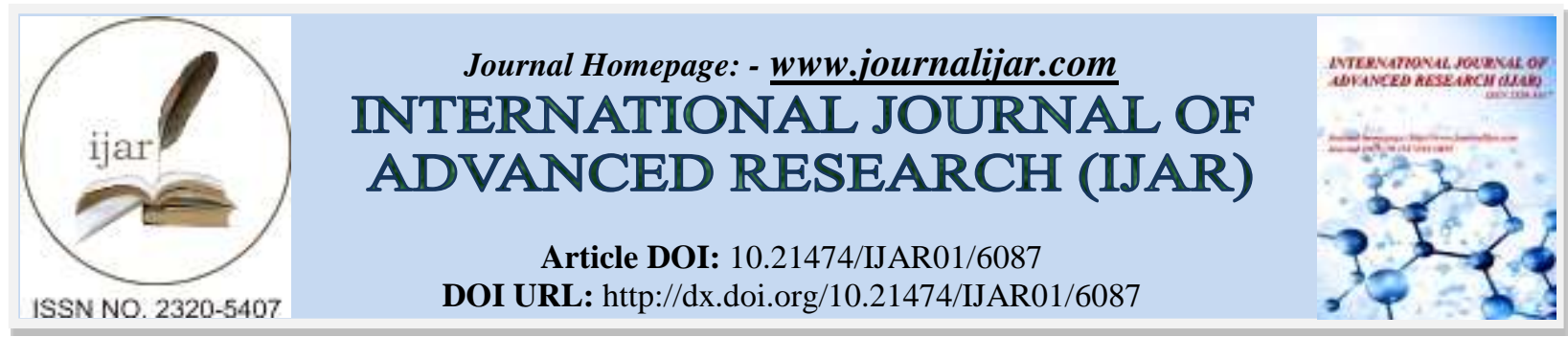

RESEARCH ARTICLE

\title{
EVALUATION AND MANAGEMENT OF DIABETIC FOOT.
}

\section{Dr. Jabreel Muzaffar ${ }^{1}$, Dr. Misbah Mehraj ${ }^{1}$ and Dr. Mohd Bilal Kaleem ${ }^{2}$.}

1. SR Department of orthopaedics SGRR Medical College, Dehradun.

2. JR Department of orthopaedics SGRR Medical College, Dehradun.

\section{Manuscript Info}

Manuscript History

Received: 17 October 2017

Final Accepted: 19 November 2017

Published: December 2017

\section{Abstract}

Diabetic foot ulcers are main cause of hospitalization in diabetic patients. Patients with diabetes mellitus are at higher risk of lower extremity complications than their non diabetic counterparts. Every year approximately 5\% of diabetic patients develop a foot ulcer. Approximately $15 \%$ of all diabetics develop foot problems during course of their illness (1-2). Diabetic foot ulcers carry a significant risk of amputation. Therefore diabetic foot disease has major medical, economic and social consequences. This was a prospective observational study conducted Tertiary Care Teaching Hospital of North India and included 100 patients with diabetic foot ulcer presenting over a period of one year. The aim of this study was to evaluate and manage different lesions of diabetic foot according to Wagner classification. A treatment oriented assessment of diabetic foot ulcers based on a cross-examination of the medical, foot, and wound history, risk factors, a systemized and detailed physical examination and the results of complementary diagnostic procedures were recorded. Diabetic foot disease presented more among male in older age group and was more common in patients with uncontrolled diabetes, with longer duration of disease, with more than one co morbid condition. 38 patients (38\%) had insulin dependent diabetics; and 87 (87\%) of them were on irregular treatment. Other 58 patients $(58 \%)$ had non-insulin dependent diabetes; out of which $19(70.0 \%)$ were on irregular. Treatment. Remaining 4 patients $(4 \%)$ were not getting any treatment for their disease The commonest disease was Grade 4 that comprised of 34 patients, followed by Grade 2 in 22 patients, followed by Grade 3 in 16 patients. These patients were managed according to Wagner classification as shown above. Conservative management with good diabetic control, antibiotic cover and foot care was carried out in 25 patients. Surgical intervention was carried out in rest of 75 patients. . The commonest procedure was incision \& drainage of foot abscess and debridement, that was performed in $40(40 \%)$ of patients; while 35 (35.0\%) patients needed some form of amputation. Multiple amputations were performed in $2(2 \%)$ patients. This study confirmed that diabetic foot ulcers is quite prevalent among diabetic population and thus, a multidisciplinary approach approach prioritizing invasive infection drainage, necrosis debridement, and the prompt start of empirical antibiotic therapy, followed by complete, appropriate

Corresponding Author:- Jabreel Muzaffar.

Address:- SR Department of orthopaedics SGRR Medical College, Dehradun. 
vascular reconstruction and patient education would be the most important tool in dealing with this major problem.

Copy Right, IJAR, 2017,. All rights reserved.

\section{Introduction:-}

Diabetic foot ulcers are main cause of hospitalization in diabetic patients. Patients with diabetes mellitus are at higher risk of lower extremity complications than their non diabetic counterparts. Every year approximately 5\%of diabetic patients develop a foot ulcer. Approximately $15 \%$ of all diabetics develop foot problems during course of their illness (1-2). Diabetic foot ulcers carry a significant risk of amputation. Therefore diabetic foot disease has major medical, economic and social consequences. It is very difficult to treat if proper protocol is not followed, resulting in longer hospital stay. Patients with diabetes have increased risk of lower extremity amputations and main cause is diabetic peripheral arterial disease accelerated by direct damage to nerves and blood vessels by high blood glucose levels. Wound healing is also impaired from affected collagen synthesis. Diabetic vascular disease has three main components : arteritis, neuropathy and large vessel atherosclerosis. the diabetic foot ulcers are often deeper and more frequently infected than other leg ulcers reflecting severe end vessel ischemia and opportunistic infection which is common affliction of diabetes. Factors such as age and duration of the disease will increase its incidence (3). Once tissue damage has occurred in form of ulceration or gangrene, aim is preservation of viable tissue. Diabetic foot ulcers are commonly classified according to Wagner classification (4). Wagner classification assesses ulcer depth and presence of osteomyelitis or gangrene.

To best of our knowledge no similar study exist from this place hence the current prospective observational study was undertaken to evaluate and manage different lesions of diabetic foot according to Wagner classification.

\section{Materials and Methods:-}

This was a prospective study conducted at SGRR Medical College, Dehradun and included 100 patients with diabetic foot ulcer presenting over a period of one year. The patients were enrolled from surgical and medical outdoor clinics, emergency units, and from other wards of the hospital. A detailed history was obtained regarding the duration of the diabetes and its type whether insulin dependent (type I) or on oral hypoglycaemic agents (typeII). They were asked about compliance and control of diabetes. A detailed history was obtained about the foot ulcers, its onset duration and progression. A detailed foot examination was performed and ulcers were classified according to Wagner's classification. (4) The Wagner classification is most commonly used for grading diabetic foot ulcers. Wagner developed a classification system and a treatment algorithm for each grade of ulcer. He asserted that ischemic index derived from Doppler flow pressures is an essential baseline test to predict ulcer healing. Vascular evaluation was performed checking capillary refill and distal pulses of the foot which included dorsalis pedis, posterior tibial, popliteal and femoral arteries. Neurological examination included light touch, pinprick, position sense and vibration sense on every patient, and data was recorded on the specified proforma.The patients were evaluated and managed by classifying their disease according to Wagner's classification for diabetic foot.(Table-1)

Table 1:- Standard Treatment of Diabetic Foot According toWagner Classification

\begin{tabular}{|c|c|c|}
\hline Grade - 0 & Foot at Risk & Prevention \\
\hline Grade - I & Localized, & $\begin{array}{l}\text { Antibiotics \& glycemic } \\
\text { superficial ulcer control }\end{array}$ \\
\hline Grade - II & $\begin{array}{l}\text { Deep Ulcer to } \\
\text { bone, } \\
\text { ligament, or } \\
\text { joint }\end{array}$ & $\begin{array}{l}\text { Debridements, } \\
\text { Antibiotics and glycemic control }\end{array}$ \\
\hline Grade - III & $\begin{array}{l}\text { Deep abscess, } \\
\text { osteomyelitis }\end{array}$ & $\begin{array}{l}\text { Debridements, some } \\
\text { form of amputation }\end{array}$ \\
\hline Grade - IV & $\begin{array}{l}\text { Gangrene of } \\
\text { toes, forefoot }\end{array}$ & Wide debridement and amputation \\
\hline Grade -V & Gangrene of enture foot & Below knee amputation \\
\hline
\end{tabular}

Statistical Analysis:-

Data was compiled and analyzed using SPSS version 16.0, and frequencies were calculated. 


\section{Results:-}

The data revealed that diabetic foot diseases affectedmales somewhat more frequently ( 66\%) as compared to females (34\%); the male to female ratio was 1.94:1. The most common age groups of diabetic patients with foot involvement were the 4 th and 5th decades. 38 patients (38\%) had insulin dependent diabetics; and $87(87 \%)$ of them were on irregular treatment. Other 58 patients $(58 \%)$ had non-insulin dependent diabetes; out of which $19(70.0 \%)$ were on irregular treatment. Remaining 4 patients $(4 \%)$ were not getting any treatment for their disease. The grade frequency of diabetic foot according to Meggitt and Wagner classification is shown in above table. The commonest disease was Grade 4 that comprised of 34 patients, followed by Grade 2 in 22 patients, followed by Grade 3 in 16 patients. (Fig. 1-10) . These patients were managed according to Wagner classification as shown above. Conservative management with good diabetic control, antibiotic cover and foot care was carried out in 25 patients. Surgical intervention was carried out in rest of 75 patients. . The commonest procedure was incision \& drainage of foot abscess and debridement, that was performed in 41(41\%) of patients; while $35(35.0 \%)$ patients needed some form of amputation. Multiple amputations were performed in 5 (5\%) patients. (Table-2-4).

\section{Discussion:-}

In the study of Viswanathan et al. (5) while evaluating post amputation outcome and associated complications in type 2 diabetic patients who had undergone major amputations in developing countries. The prevalence of minor amputation and foot deformity was high among Bangladesh population. Recurrence of foot ulceration was more in Tanzania (30\%) than in India (9\%) and

Table 2:- Epidemiological Profile of Study Population

\section{Parameters Variables}

Male to Female ratio

Common Age Groups

Urban Vs Rural

Smoking Vs Non Smoker

Alcohol Vs Non Alcoholics

Co-morbid conditions Rate

Infected Vs Non infected

Normal weight Vs Overweight Vs Underweight

PVD Vs No PVD

Glycaemic control : Poor Vs Fair Vs Good

\section{On Insulin Treatment Vs On OHA V}

Not getting any treatment

\subsection{4:1}

4th and 5th decades

$65(65 \%)$ Vs $35(35 \%)$

29(29\%) Vs 71(71\%)

$12(12 \%)$ Vs $88(88 \%)$

$37(\%)$

09(09\%) Vs 91(91\%)

59(59\%) Vs39(39)\%Vs2(2\%)

$8(8 \%)$ Vs $92(92 \%)$

$79(79 \%)$ Vs $15(15 \%)$ Vs 6(6\%)।

$38(38 \%)$ Vs58(58\%) Vs4( 4\%)

Duration of diabetes in years >5 years Vs < 5years 67(67\%) Vs 33(33\%)

Table 3:- Clasification of Diabetic Foot Study Population

Wagner Classification $\mathbf{N}(\%)$

Grade-0 0(0\%)

Grade -I 18(18\%)

Grade-II 22(22\%)

Grade-III 16(16\%)

Grade -IV 34(34\%)

Grade-V 10(10\%) 
Table 4:- Management of Diabetic Foot Study Populati Treatment No. of Patients (\%)

Conservative $25(25 \%)$

Surgical $75(75 \%)$

Incision and drainage 4(4\%)

Debridement 36(36\%)

Amputations 35(35\%)

Ray's $4(4 \%)$

Transmetatarsal 7(7\%)

Below Knee Amputation 12(12\%)

Above Knee Amputation 10(10\%)

Multiple 2 amputations(2\%)

\section{Discussion:-}

In the study of Viswanathan et al. (5) while evaluating post amputation outcome and associated complications in type 2 diabetic patients who had undergone major amputations in developing countries .The prevalence of minor amputation and foot deformity was high among Bangladesh population. Recurrence of foot ulceration was more in Tanzania (30\%) than in India (9\%) and Bangladesh (11\%). Re-amputation rate was similar in all groups (3\%). The use of artificial limb was most in Bangladesh (97\%). The causes of death were infection due to septicemia and cardiovascular events which finally led to multisystem organ failure The results of the current study are in accordance with the study of Ali SM et al (6) as there were 65\% males and 35\% females. Ninety nine patients were type II diabetics, $38 \%$ were either smokers or had other addictions (or were addicted to tobacco). Awareness about risk factors causing foot problems was lacking among all patients. Fifty percent patients were on oral hypoglycaemic agents, $48 \%$ were insulin treated, while $2 \%$ were on diet and exercise alone. Glycaemic control was poor in $70 \%$, fair in $16 \%$ and was good in $14 \%, 31 \%$ were overweight and $5 \%$ patients were underweight. Duration of diabetes was greater than ten years in $58 \%$, toes were affected in $44 \%$, sole/metatarsal in $18 \%$; rest included malleoli, heel etc. Eleven patients had ulcers on both feet. Neuropathic ulcer were $42 \%$, neuroischaemic $58 \%$, Sixty nine percent patients were in the age group between 40-60 years. Cause unknown $29 \%$ blisters and boil $14 \%$, trauma/cutting $17 \%$, burns $8 \%$, dry skin/callus . $10 \%$. Fundal changes were present in $37 \%$; proteinuria in $37 \%$, ischaemic heart disease in $20 \%$, hypertension $18 \%$, In $60 \%$ more than one antibiotic was used. Foot ulcers of fifty nine patients healed on conservative management, six patients had below knee amputation, fifteen had toe amputation; nine were still on treatment, eleven lost contact. In the study of Viswanathan et al (7) while determining the prevalence of foot complications such as neuropathy, peripheral vascular disease (PVD), amputations and infections and the associated diabetic complications and practice of foot care among these subjects reported that the prevalence of infection was $6-11 \%$ and prevalence of amputation was 3\% in type 2 diabetic patients. Neuropathy (15\%) was found to be an important risk factor for diabetic foot infections. Further study suggested that effective foot care advice should be propagated to reduce the burden imposed by diabetic foot complication particularly in developing countries like India. In the study of Ahmad Wet al (3) male dominated the study population with majority in the age range of 4070) years. Right foot was more commonly involved (65.3\%). $91.3 \%$ patients had diabetes of more than 5 years duration. No treatment had been received by $47.4 \%$ patients while $41.3 \%$ were on oral anti-diabetics; $11.2 \%$ patients were on insulin. All patients had type 2 diabetes mellitus. Neuropathy was present in 51\% patients, $62.8 \%$ had absent or diminished peripheral pulses, $43.4 \%$ had poorly controlled diabetes. According to the Wagner classification $30.6 \%$ patients had grade 1, 26.5\% had grade 2, and $42.9 \%$ had grade 3 diabetic foot. Evidence of infection was seen in $85.7 \%$ patients: staphylococcus aureus was isolated in $43.4 \%$ patients. Osteomyelitis was present in $42.9 \%$ patients. Surgical intervention was performed in $85.7 \%$ patients. Direct relation was found between the duration of diabetes, sugar control, peripheral neuropathy, peripheral arterial disease. grade of diabetic foot, evidence of osteomyelitis, intervention and the outcome of the disease .In view of the results of the current study, we stress the need for a clinical diagnosis of diabetic foot ulcers.

Regarding treatment, we propose a multidisciplinary approach prioritizing invasive infection drainage, necrosis debridement, and the prompt start of empirical antibiotic therapy, followed by complete and appropriate vascular reconstruction. For severe Diabetic foot ulcers, we suggest that negative pressure wound therapy (NPWT) be included in the treatment pathway. We also provide rules for managing particular situations, such as osteomyelitis. 


\section{Conclusion:-}

This study also confirmed that diabetic foot ulcers is quite prevalent among diabetic population and thus, a multidisciplinary approach approach prioritizing invasive infection drainage, necrosis debridement, and the prompt start of empirical antibiotic therapy, followed by complete, appropriate vascular reconstruction and patient education would be the most important tool in dealing with this major problem.

\section{References:-}

1. Boulton AJ, Vileikyte L, Ragnarson-Tennvall G, ApelqvistJ, The Global Burden of Diabetic foot disease. Lancet 2005;366:1719-21

2. Shearman CP, Widhaber R. Foot Complications in patients with Diabetes. Surgery 2010; 28:288-92

3. Ahmad W, Khan IA, Ghaffar S, Al-Swailmi FK, Khan I.Risk factors for diabetic foot ulcer. J Ayub Med Coll Abbottabad2013;25(1-2):16-8

4. Mugambi-Nturibi E, Otieno CF, Kwasa TO, Oyoo GO,Acharya K. Stratification of persons with diabetes into

5. risk categories for foot ulceration. East Afr Med J 2009;86:233-9.

6. Viswanathan V1, Wadud JR, Madhavan S.Comparison of post amputation outcome in patients with type 2 diabetes from specialized foot care centres in three developing countries. Diabetes Res Clin Pract 2010;88(2):146-50

7. Ali SM1, Basit A, Sheikh T, Mumtaz S, Hydrie MZ. Diabetic foot ulcer--a prospective study. J Pak Med Assoc 2001;51(2):78-81

8. Viswanathan V1, Thomas N, Tandon N, et al. Profile of diabetic foot complications and its associated complications--a multicentric study from India. J Assoc Physicians India 2005;53:933-6 